\title{
DUBA-UBR5 axis: other than transactivation
}

\author{
Cell Research (2015) 25:273-274. doi:10.1038/cr.2015.13; published online 30 January 2015
}

The genomic and transcriptomic analysis has led to the identification of transcriptional networks that affect the differentiation of Th17 cells, while the protein-protein networks and the posttranslational modifications that control the development of Th17 cells have not been established yet. A recent publication by Rutz et al. in Nature describes a novel deubiquitylating enzyme DUBA as a negative regulator for IL-17 by regulating stability of ROR $\gamma \mathrm{t}$, the master transcription factor that induces Th17 cells.

IL-17-producing $\mathrm{CD}^{+}{ }^{+} \mathrm{T}$ cells have been described to be critical for inducing various pathological conditions including autoimmune diseases like psoriasis, inflammatory bowel disease and multiple sclerosis. Th17 cells produce not only IL-17 but also a number of other cytokines including IL-17A, IL-17F, TNF- $\alpha$, IL-22 and IL-21 [1, 2]. However, as one of the dominant cytokines, IL-17 has been described to have multiple proinflammatory functions and has been shown to induce tissue inflammation in different disease models [3]. Although a number of groups have reported the detailed transcriptomic analysis of Th17 cells, the protein-protein network that governs the development of Th17 cells has not been established. Rutz et al. [4] now report a novel deubiquitlylating enzyme DUBA, which plays a critical role in IL-17 production.

By generating the DUBA-deficient mice, the authors discovered that DUBA negatively regulates IL-17 production not only in the $\mathrm{CD} 4^{+} \mathrm{T}$ cells but also in the type 3 innate lymphoid cells (ILC3), while the other $\mathrm{T}$ cell subsets Th1 and Th2 were found to have normal levels of IL-17 in the DUBA-deficient mice. $\mathrm{ROR} \gamma \mathrm{t}$ is the master transcription factor that transactivates IL-17 in both Th17 and ILC3 cells. In vivo activation of T cells by injecting anti-CD3 antibodies resulted in enhanced Th17 response with more severe inflammation in the small intestine in the DUBA-deficient mice. Foxp $3^{+}$Treg cells, which have a reciprocal relationship with Th17 cells, also expressed more ROR $\gamma \mathrm{t}$ and produced more IL-17 in the DUBAdeficient mice, further supporting the potential repression of IL-17 by DUBA.

Differentiation of Th17 cells can be induced by different culture conditions. The combination of exogenous TGF- $\beta 1$ and IL- 6 induces Th17 cells that co-produce IL-17 and IL-10 and are generally nonpathogenic in terms of induction of autoimmune diseases. The Th17 cells induced under another condition with IL-1 $\beta$, IL- 6 and IL-23 produce IL-17 and IFN- $\gamma$, and these Th17 cells are highly pathogenic and induce potent autoimmune diseases upon adoptive transfer. Interestingly, the most significant function of DUBA on IL-17 production appears only in the presence of TGF- $\beta$, which generates nonpathogenic Th17 cells. In the presence of TGF- $\beta$, the half-life of ROR $\gamma \mathrm{t}$ was maintained over $6 \mathrm{~h}$ in DUBA-knockout (KO) Th17 cells while its half-life in wild-type (WT) Th17 cells only lasted for $1 \mathrm{~h}$. These data suggest that in the presence of TGF- $\beta$, DUBA inhibits ROR $\gamma \mathrm{t}$ by mostly destabilizing ROR $\gamma$ t protein. By performing mass spectrometry between WT and DUBA-KO Th17 cells, the authors narrowed down DUBA substrates. Further short interfering RNA (siRNA) screening identified a ubiquitin ligase UBR5 as an interacting protein with
DUBA and its depletion led to increased IL-17A production and ROR $\gamma$ t expression in Th17 cells. Biochemical analysis suggests that ubiquitin ligase UBR5 is stabilized by DUBA. The authors therefore hypothesize that TGF- $\beta$ stimulation enhances the activity of UBR5 which is stabilized by DUBA, and two together then target ROR $\gamma$ t for proteosomal degradation.

These results are consistent with one recent study reporting that $\mathrm{ROR} \gamma \mathrm{t}$ protein is modified by $\mathrm{K} 48$-linked ubiquitylation and undergoes proteasomemediated degradation. Another protein USP17 was shown as a deubiquitinase for ROR $\gamma t$, which stabilizes ROR $\gamma \mathrm{t}$ expression and promotes the function of Th1 7 cells [5]. Rutz et al. [4] identified the enzyme which mediates opposite function in that it induces reversible covalent modification of ROR $\gamma \mathrm{t}$. UBR5 was demonstrated to promote ROR $\gamma \mathrm{t}$ ubiquitylation and target it for proteosomal degradation. It will be interesting to further investigate whether UBR5 antagonizes the activity of USP17, whether USP17 activity is induced by TCR or TGF- $\beta$ signaling and how UBR5 and USP17 coordinately act to induce or inhibit Th17 differentiation and expansion.

Many deubiquitinases are found to associate with ubiquitin ligases, but the interaction between opposing catalytic activities may lead to diverse effects, including tuning the ubiquitylation status of the target proteins, modulating signaling pathways, or editing the ubiquitin chains on the substrate [6]. In the paper by Rutz et al. [4], the coupling between the deubiquitinase DUBA and the ubiquitin ligase UBR5 serves another purpose. The associa- 
tion of DUBA stabilizes UBR5 and reciprocally, UBR5 destabilizes DUBA through ubiquitylation. The stabilization of ubiquitin ligases is a major aspect of deubiqutinase physiology and this occurs in many ligases, which have the tendency to undergo degradative auto-ubiquitylation like UBR5. The ultimate effect of DUBA in this case is to maintain URB5 and destabilize the substrate protein ROR $\gamma \mathrm{t}$.

DUBA was previously reported to inhibit the production of type I IFN in innate cells by removing K63-linked ubiquitin chain on TRAF3 [7, 8]. The new observation made by authors suggests that DUBA expressed in T cells participates in the regulation of $\mathrm{T}$ cells to limit IL-17 cytokine production. Therefore, it emphasizes a negative role of DUBA in the immune system, suggesting that DUBA is a key factor in restraining inflammation by specifically limiting Th17 (but not Th1 or Th2) responses. Since deubiquitinase inhibitors have been widely used as drugs in the clinical treatment, DUBA provides a potential therapeutic target for human diseases by regulating activity of Th17 cells.

Mechanistically, in addition to the recognition of K63-linked ubiquitin chain [8], DUBA was also shown to be involved in K48-linked ubiquitin chain cleavage, different from other OTU domain-containing deubiquitinases. Moreover, DUBA affects ROR $\gamma \mathrm{t}$ and IL-17 only in nonpathogenic Th17 cells induced by TGF- $\beta$, but not in pathogenic Th17 cells induced by IL$1 \beta$ stimulation. In the previous study, DUBA protein was increased in the absence of IL-1R1 signaling in BMDCs [7]. It is conceivable that the downregulation of DUBA under IL-1R1 signaling may also occur in T cells, leading to loss of activity of DUBA during pathogenic IL-1 $\beta$ differentiation conditions, which results in the generation of highly potent pathogenic Th17 cells. The question remains, whether overexpression of DUBA in pathogenic Th17 cells will convert them into a nonpathogenic Th17 state.

In the modulation of the balance between Treg and Th17 cells, HIF-1 $\alpha$ was shown to favor Th17 differentiation over Tregs by promoting Foxp3 protein degradation via polyubiquitylaiton [9]. The paper by Rutz et al. [4] highlighted a similar regulation in $\mathrm{ROR} \gamma \mathrm{t}$ protein degradation, which is mediated by DUBA and UBR5. The finding of ubiquitylation of ROR $\gamma t$ has expanded our understanding of the dynamic nature of ROR $\gamma \mathrm{t}$ protein in the stabilization of Th17 cell subset at the posttranslational level. It is not clear which specific lysine residues on ROR $\gamma t$ protein are modified by ubiquitylation and whether other posttranslational modifications help to coordinate ROR $\gamma \mathrm{t}$ stability and function. More studies on the spatial and temporal regulation of DUBA and UBR5 activity under different microenvironments and differentiation conditions can help exploit this pathway for therapeutic ends.

\section{Chuan $\mathrm{Wu}^{1}$, Zuojia Chen ${ }^{1}$, Vijay K Kuchroo $^{1}$}

${ }^{1}$ Evergrande Center for Immunologic Diseases, Harvard Medical School, Brigham and Women's Hospital, Boston, MA 02115, USA

Correspondence: Vijay K Kuchroo

E-mail: vkuchroo@rics.bwh.harvard.edu

\section{References}

1 Harrington LE, Hatton RD, Mangan PR, et al. Nat Immunol 2005; 6:1123-1132.

2 Park H, Li Z, Yang XO, et al. Nat Immunol 2005; 6:1133-1141.

3 Korn T, Bettelli E, Oukka M, et al. Annu Rev Immunol 2009; 27:485-517.

4 Rutz S, Kayagaki N, Phung QT, et al. Nature 2014 Dec 3. doi: 10.1038/nature13979

5 Han L, Yang J, Wang X, et al. J Biol Chem 2014; 289:25546-25555.

6 Komander D, Clague MJ, Urbe S. Nat Rev Mol Cell Biol 2009; 10:550-563.

7 González-Navajas JM, Law J, Nguyen KP, et al. J Exp Med 2010; 207:2799-2807.

8 Kayagaki N, Phung Q, Chan S, et al. Science 2007; 318:1628-1632.

9 Dang EV, Barbi J, Yang HY, et al. Cell 2011; 146: $772-784$ 